\title{
LAW OF LARGE NUMBERS FOR DISCRETELY OBSERVED RANDOM FUNCTIONS
}

\author{
STANISLAV NAGY ${ }^{1,2}$ AND IRÈNE GIJBELS ${ }^{1}$
}

\begin{abstract}
A strong law of large numbers for continuous random functions, and associated tensor product surfaces is established in the setup of discretely observed functional data. The result is shown in the framework of uniform convergence of functions, and stated without imposing any distributional assumptions. It is demonstrated that, under mild conditions, laws of large numbers for continuously observed functional data imply the corresponding laws under the discrete observational design of functions. Applications to the problem of estimation of expectation functions and covariance surfaces for discretely observed functional data are discussed.
\end{abstract}

Key Words And Phrases: consistency, functional data, functional moments, strong law of large numbers.

\section{INTRODUCTION}

The strong law of large numbers (SLLN) for Banach-valued random variables has attracted a great deal of attention in the probabilistic literature (cf Ledoux and Talagrand, 2011). One of its most important applications in statistics comes from functional data analysis (Ramsay and Silverman, 2005). This, however, hinges on the crucial assumption that all the involved random functions are observed completely, i.e. their functional values are known at each point of their domain. This is often not the case in practice. Usually, functional data are observed only at a finite number of discrete time points, possibly different for each random function. Subsequently, the discrete realizations of the random functions are preprocessed to constitute elements of the space of continuous functions, and only then data analysis is applied to these reconstructed curves.

Here, we study SLLN in the latter setting of discretely observed functional data. We state conditions under which a SLLN can be established, and relate these results with the general SLLN for Banach-valued data (Ledoux and Talagrand, 2011, Corollary 7.10). An important finding is that under mild conditions, the general Kolmogorov's SLLN for completely observed random functions directly implies the SLLN for discretely observed functional data.

Related results for discretely sampled (dense and sparse) functional data have already been studied in the literature; see, for instance, Cai and Yuan (2011) and references therein. However, in that research, random functions are assumed to come from certain classes of Hilbert-Sobolev, or Hilbert spaces. Thus, the data curves are bound to be smooth, integrable, or a combination of these, which is not always the case. Moreover,

\footnotetext{
1 KU Leuven, Department of Mathematics and Leuven Statistics Research Centre (LStat), Belgium

2 Charles University, Department of Probability and Math. Statistics, Czech REPUBLIC

E-mail addresses: stanislav.nagy@kuleuven.be.

Date: February 13, 2017.
} 
continuous functions usually do not form a closed subspace in these functional spaces, introducing additional theoretical complications. In the present paper, we consider random variables coming from the Banach space of continuous functions over a compact interval, without imposing further distributional assumptions.

In Section 2 the definitions and the notation are introduced. In Section 3, the SLLN for discretely observed functions is stated, with some of its applications discussed in Section 4. In the Appendix the proofs of the theoretical results can be found.

\section{Notation And Preliminaries}

Denote by $(\Omega, \mathcal{F}, \mathrm{P})$ the probability space on which all the random variables are defined. For a measurable space $S$, write $\mathcal{P}(S)$ for the set of all probability measures on $S$. For $P \in \mathcal{P}(S), X \sim P$ denotes an $S$-valued random variable $X$ distributed according to $P$.

Assume that $d \in \mathbb{N}$ is given. For the $d$-dimensional unit cube

$$
[0,1]^{d}=\left\{t=\left(t_{1}, \ldots, t_{d}\right): t_{j} \in[0,1] \text { for all } j=1, \ldots, d\right\}
$$

denote the space of $\mathbb{R}$-valued continuous functions over $[0,1]^{d}$ by $\mathcal{C}\left([0,1]^{d}\right)$ and endow it with the usual uniform norm

$$
\|x\|=\sup _{t \in[0,1]^{d}}|x(t)| \text { for } x \in \mathcal{C}\left([0,1]^{d}\right) .
$$

For $x \in \mathcal{C}\left([0,1]^{d}\right)$ and $\delta>0$, define the (minimal) modulus of continuity of $x$ at $\delta$ as

$$
\mathcal{M}(x, \delta)=\sup _{s, t:\|s-t\|_{E}<\delta}|x(s)-x(t)| .
$$

The notation $\|\cdot\|_{E}$ stands for the Euclidean norm in $\mathbb{R}^{d}$.

For a function $x \in \mathcal{C}([0,1]) \equiv \mathcal{C}\left([0,1]^{1}\right)$ and $d \geq 1$, the $d$ th tensor product of $x$ is the mapping from $\mathcal{C}([0,1])$ into $\mathcal{C}\left([0,1]^{d}\right)$ given by

$$
\bigotimes_{j=1}^{d} x:[0,1]^{d} \rightarrow \mathbb{R}:\left(t_{1}, \ldots, t_{d}\right) \mapsto \prod_{j=1}^{d} x\left(t_{j}\right) .
$$

Evidently $\bigotimes_{j=1}^{1} x \equiv x$. The second tensor product of $x$ will be denoted also by $x \otimes x$. Note that (2) is a special case of the usual tensor product of $d$ functions $\left\{x_{j}\right\}_{j=1}^{d} \subset \mathcal{C}([0,1])$ defined by

$$
\bigotimes_{j=1}^{d} x_{j}:[0,1]^{d} \rightarrow \mathbb{R}:\left(t_{1}, \ldots, t_{d}\right) \mapsto \prod_{j=1}^{d} x_{j}\left(t_{j}\right)
$$

Extensions of the results provided here to the setup of general tensor products is straightforward. Though, for simplicity, we consider only products of the form (2), keeping in mind the motivation given by applications in functional data analysis.

In this paper, we provide a SLLN for random functions $X \sim P \in \mathcal{P}(\mathcal{C}([0,1]))$ and, more generally, a SLLN for estimation of tensor product surfaces given by

$$
\mathrm{T}=\mathrm{E}\left[\bigotimes_{j=1}^{d} X\right]
$$

In (3), the expectation of the random variable $\bigotimes_{j=1}^{d} X$ is taken in the sense of Bochner (strong) integral in the Banach space $\mathcal{C}\left([0,1]^{d}\right)$ (for integrals of Banach-valued random 
variables, see Ledoux and Talagrand, 2011, Section 2.1). If T exists, then for each $t \in$ $[0,1]^{d}$ its value is given by $\mathrm{T}(t)=\mathrm{E}\left[\bigotimes_{j=1}^{d} X(t)\right]$.

In functional data analysis, various versions of theoretical results involving random curves can be distinguished. These depend on the observational design of the data curves, which in most cases takes one of the following forms:

- continuous (or complete) design: in this setting random functions are observed at each time point $t \in[0,1]$, or

- discrete design: in this setting the values of random functions are observed only in a finite number of points in the domain.

In the former case, each functional value $X(t) \in \mathbb{R}$ is supposed to be known to the experimenter. In current statistical literature, this is usually considered standard (Ramsay and Silverman, 2005, Ferraty and Vieu, 2006), and most theoretical results are stated for continuously observed random functions.

In practice, however, the continuous design setting often turns out to be unrealistic, as data are observed discretely. In nonparametric data analysis, the random curves are then bound to be preprocessed to form elements of $\mathcal{C}([0,1])$. In the sequel, assume that this is the case.

For a generic function $X$, denote the cardinality of the set of its distinct observed values by $M=1,2, \ldots$, and denote the time points at which these values are known by

$$
\mathcal{T}=\left(T_{1}, \ldots, T_{M}\right) \in[0,1]^{M}, \text { where } 0 \leq T_{1}<T_{2}<\cdots<T_{M} \leq 1 .
$$

The quantities $M$ and $\mathcal{T}$ may be considered random, without loss of generality. Note that the time points for each curve (4) are assumed to be ordered increasingly in magnitude. When needed, append the vector $\mathcal{T}$ with $T_{0}=0$ and $T_{M+1}=1$. For a curve $X$ we are then provided with the vector

$$
X(\mathcal{T})=\left(X\left(T_{1}\right), \ldots, X\left(T_{M}\right)\right) \in \mathbb{R}^{M} .
$$

To perform functional data analysis, reconstruction of $X$ using observation points (5) can be made by approximation, or interpolation, of points (5) at (4). This way we obtain for each set of observation points a single curve in $\mathcal{C}([0,1])$, further denoted by a tilde as in $\tilde{X}$, serving as a continuous representative of the unobservable curve $X$.

If a random sample $X_{1}, \ldots, X_{n}$ is observed from distribution $P \in \mathcal{P}(\mathcal{C}([0,1]))$ within the discrete design setting, vector (4) associated with $X_{i}$ will be designated by a further subscript $i$, i.e. $M_{i}$ for the size of the vector $\mathcal{T}_{i}$, whose $j$ th element is denoted by $T_{j, i}$.

Data imputation as described above can be reliable only if the reconstruction procedure provides an accurate approximation of the original data. Therefore, we make use of the following assumptions, under which it turns out to be possible to formulate a SLLN:

- independence of observation points and random curves random vector $\mathcal{T}_{n}$ is independent of $X_{n}$ for all $n \in \mathbb{N}$,

- concentration of observation points

$$
\nu\left(\mathcal{T}_{n}\right)=\max _{j=0, \ldots, M_{n}}\left|T_{j+1, n}-T_{j, n}\right| \underset{n \rightarrow \infty}{\stackrel{\text { a.s. }}{\longrightarrow}} 0,
$$


- approximation error bound for tensor surfaces

there exist $C, D>0$ such that for any $x \in \mathcal{C}([0,1])$ observed at $\mathcal{T}$

(D.3)

$$
\left\|\bigotimes_{j=1}^{d} x-\bigotimes_{j=1}^{d} \widetilde{x}\right\| \leq D \mathcal{M}\left(\bigotimes_{j=1}^{d} x, C \nu(\mathcal{T})\right) \text {. }
$$

Conditions (D.1) and (D.2) impose restrictions on the observational design of functional data; in what follows, we shall refer to random functions observed under (D.1) and (D.2) as to densely discrete (or simply dense) functional data (cf Li and Hsing, 2010). Condition (D.1) is standard and usually can be assumed to be satisfied. Condition $(\mathbf{D} .2)$ can be verified if each vector $\mathcal{T}_{n}$ corresponds to a random sample as described in the following lemma.

Lemma 1. For each $n \in \mathbb{N}$ let $\mathcal{T}_{n}$ be a realization of a random sample of random size $M_{n}$, $M_{n} \underset{n \rightarrow \infty}{\stackrel{\text { a.s. }}{\longrightarrow}} \infty$, from a distribution on $[0,1]$ having density $f$ such that $\inf _{t \in[0,1]} f(t)>0$. Then condition (D.2) is satisfied for $\left\{\mathcal{T}_{n}\right\}_{n=1}^{\infty}$.

Condition (D.3) deals with the approximation of random functions and their tensor products, and can be shown to be satisfied, for instance, for piecewise (multi-)linear interpolation.

Lemma 2. For any $x \in \mathcal{C}([0,1])$ observed at $\mathcal{T}$, consider the interpolating function $\widetilde{x}$ defined as follows

$$
\widetilde{x}(t)= \begin{cases}x\left(T_{1}\right) & \text { for } t \in\left[0, T_{1}\right), \\ \frac{x\left(T_{j+1}\right)-x\left(T_{j}\right)}{T_{j+1}-T_{j}}\left(t-T_{j}\right)+x\left(T_{j}\right) & \text { for } t \in\left[T_{j}, T_{j+1}\right), j=1, \ldots, M-1, \\ x\left(T_{M}\right) & \text { for } t \in\left[T_{M}, 1\right] .\end{cases}
$$

Then, for any $d \in \mathbb{N}$, condition $(\mathbf{D . 3})$ is satisfied with $C=\sqrt{d}$ and $D=1$.

For $d=1$ Nagy et al. (2016) used piecewise linear interpolation as described in Lemma 2 to obtain continuous versions of densely observed curves. Therein, it is shown that basing statistical inference on a sample of curves $\widetilde{X}_{1}, \ldots, \widetilde{X}_{n}$ instead of $X_{1}, \ldots, X_{n}$, Varadarajan's type of result (Varadarajan, 1958) for the empirical measure of the random sample of reconstructed curves can be obtained. As a corollary, under suitable assumptions, pointwise convergence $\mathrm{E}\left[\widetilde{X}_{n}(t)\right] \underset{n \rightarrow \infty}{\longrightarrow} \mathrm{E}[X(t)]$, and a pointwise SLLN $\sum_{i=1}^{n} \widetilde{X}_{i}(t) / n \underset{n \rightarrow \infty}{\stackrel{\text { a.s. }}{\longrightarrow}} \mathrm{E}[X(t)]$ for each fixed $t \in[0,1]$ were shown. In the present paper, we extend these results to hold in the sense of uniform convergence of functions, that is to obtain convergence in the space of functions $\mathcal{C}([0,1])$ with norm $(1)$.

By considering the tensor product $\bigotimes_{j=1}^{d} \widetilde{x}$ of a curve $x$ interpolated by $(6)$ for $d>1$, the so-called multi-linear interpolation of the tensor product surface $\bigotimes_{j=1}^{d} x$ takes place. This concept is well-known in multivariate interpolation theory, and can be shown to have many beneficial theoretical properties (cf de Boor 2001, Chapter XVII for exposition, Klimke and Wohlmuth 2005 for recent advances in the topic, or Abramowicz and Seleznjev 2013 for an application in probability). 


\section{SLLN for Tensors of Discretely Observed Functions}

In this section a SLLN for tensor surfaces of densely observed functional data is presented. To this end, assume that we are given a random sample $X_{1}, X_{2}, \ldots, X_{n}$ of functions from distribution $P \in \mathcal{P}(\mathcal{C}([0,1]))$. A reasonable estimator of the surface $\mathrm{T}$ from $(3)$ in the complete observational design setting is certainly the mean function

$$
\mathrm{T}_{n}(t)=\frac{1}{n} \sum_{i=1}^{n} \bigotimes_{j=1}^{d} X_{i}(t) \text { for } t \in[0,1]^{d} .
$$

In a densely discrete design setting, it is natural to plug the reconstructed curves into $\mathrm{T}_{n}$ and consider an estimator

$$
\widetilde{\mathrm{T}}_{n}(t)=\frac{1}{n} \sum_{i=1}^{n} \bigotimes_{j=1}^{d} \widetilde{X}_{i}(t) \text { for } t \in[0,1]^{d} .
$$

For $\mathrm{T}_{n}$, the classical SLLN for Banach-valued random variables (Ledoux and Talagrand, 2011 , Corollary 7.10$)$ can be applied in the space $\mathcal{C}\left([0,1]^{d}\right)$. Still, in spaces of continuous functions, alternative sets of assumptions for a SLLN to hold have been proposed in the literature. Here we mention the work of Hansen $(1982,2012)$, who proved a SLLN for continuous functional data using the notion of moment continuity. The following definition provides an extension of this concept to tensor surfaces of random functions.

Definition. For $d \in \mathbb{N}$ we say that $X \sim P \in \mathcal{P}(\mathcal{C}([0,1]))$ is $d$ th order moment continuous, denoted by $X \in \mathcal{M C}_{d}$, if

$$
\lim _{\delta \rightarrow 0+} \mathrm{E}\left[\sup _{s:\|s-t\|_{E}<\delta}\left|\bigotimes_{j=1}^{d} X(s)-\bigotimes_{j=1}^{d} X(t)\right|\right]=0 \quad \text { for all } t \in[0,1]^{d} .
$$

Notice that the supremum is taken inside the expectation; this fact is essential in what follows. As we show in the next theorem containing the main contribution of the paper, the conditions under which a SLLN holds true for complete and dense functional data, are virtually identical.

Theorem 1. Let conditions (D.1)-(D.3) be satisfied for the sampling design of the random curve $X \sim P \in \mathcal{P}(\mathcal{C}([0,1]))$. Then the following statements are equivalent

(i) $\mathrm{E}\left[\left\|\bigotimes_{j=1}^{d} X\right\|\right]<\infty$,

(ii) $X \in \mathcal{M C}_{d}$ and $\mathrm{E}\left[\left|\bigotimes_{j=1}^{d} X\left(t_{0}\right)\right|\right]<\infty$ for some $t_{0} \in[0,1]^{d}$,

(iii) $\left\|\mathrm{T}_{n}-\mathrm{T}\right\| \underset{n \rightarrow \infty}{\stackrel{\text { a.s. }}{\longrightarrow}} 0$.

If any of the above statements hold true, then also

(iv) $\left\|\widetilde{\mathrm{T}}_{n}-\mathrm{T}\right\| \underset{n \rightarrow \infty}{\stackrel{\text { a.s. }}{\longrightarrow}} 0$.

Note that as part of the statement of Theorem 1 we obtain also the existence of the expectation (3).

The assumption of compactness of the domain $[0,1]$ in Theorem 1 is essential — without this condition, Part $(i)$ does not imply the other statements of the theorem. For a noncompact bounded domain $U \subset \mathbb{R}$, a variant of Theorem 1 can be recovered if random functions $X=X(\omega)$ are bounded and uniformly continuous on $U$, for almost all $\omega \in \Omega$. 
In that case, one can apply Theorem 1 to the (unique) continuous extension of $X$ to the closure of $U$, which is a compact set in $\mathbb{R}$.

A simple relation between the equivalent statements of the previous theorem for various values of $d$ can be established.

Theorem 2. Let $d \in \mathbb{N}$ and $X \sim P \in \mathcal{P}(\mathcal{C}([0,1]))$. If $\mathrm{E}\left[\left\|\bigotimes_{j=1}^{d} X\right\|\right]<\infty$, then also $\mathrm{E}\left[\left\|\bigotimes_{j=1}^{d^{\prime}} X\right\|\right]<\infty$ for any $d^{\prime}=1,2, \ldots, d-1$.

\section{Applications to Mean Functions and Covariance Surfaces}

For $d=1$, the following corollary of Theorem 1 for the expectation of random functions is immediate.

Theorem 3. Let conditions (D.1) $-(\mathbf{D} .3)$ be satisfied for $d=1$ for the sampling design of the random curve $X \sim P \in \mathcal{P}(\mathcal{C}([0,1]))$. Then the following statements are equivalent

(i) $\mathrm{E}[\|X\|]<\infty$,

(ii) $X \in \mathcal{M C}_{1}$ and $\mathrm{E}\left[\left|X\left(t_{0}\right)\right|\right]<\infty$ for some $t_{0} \in[0,1]$,

(iii) $\left\|1 / n \sum_{i=1}^{n} X_{i}-\mathrm{E}[X]\right\| \underset{n \rightarrow \infty}{\stackrel{\text { a.s. }}{\longrightarrow}} 0$.

If any of the above statements hold true, then also

(iv) $\left\|1 / n \sum_{i=1}^{n} \tilde{X}_{i}-\mathrm{E}[X]\right\| \underset{n \rightarrow \infty}{\stackrel{\text { a.s. }}{\longrightarrow}} 0$.

The covariance surface of the random function $X$ can be defined as an infinite-dimensional extension of the covariance matrix

$$
\sigma(t)=\mathrm{E}\left[\bigotimes_{j=1}^{2}(X-\mathrm{E}[X])(t)\right] \text { for } t \in[0,1]^{2},
$$

provided that the expectation exists. The surface $\sigma$ can then be estimated within the complete, and the densely discrete design setting, respectively, by

$$
\begin{aligned}
& \sigma_{n}(t)=\frac{1}{n} \sum_{i=1}^{n}\left(X_{i}\left(t_{1}\right)-\frac{1}{n} \sum_{j=1}^{n} X_{j}\left(t_{1}\right)\right)\left(X_{i}\left(t_{2}\right)-\frac{1}{n} \sum_{j=1}^{n} X_{j}\left(t_{2}\right)\right), \\
& \widetilde{\sigma}_{n}(t)=\frac{1}{n} \sum_{i=1}^{n}\left(\widetilde{X}_{i}\left(t_{1}\right)-\frac{1}{n} \sum_{j=1}^{n} \widetilde{X}_{j}\left(t_{1}\right)\right)\left(\widetilde{X}_{i}\left(t_{2}\right)-\frac{1}{n} \sum_{j=1}^{n} \widetilde{X}_{j}\left(t_{2}\right)\right),
\end{aligned}
$$

both defined for $t=\left(t_{1}, t_{2}\right) \in[0,1]^{2}$. The following theorem is a direct consequence of Theorems 1 and 2 .

Theorem 4. Let conditions (D.1)-(D.3) be satisfied for $d=2$ for the sampling design of the random curve $X \sim P \in \mathcal{P}(\mathcal{C}([0,1]))$. Then the following statements are equivalent

(i) $\mathrm{E}[\|X \otimes X\|]<\infty$,

(ii) $X \in \mathcal{M C}_{2}$ and $\mathrm{E}\left[\left|X \otimes X\left(t_{0}\right)\right|\right]<\infty$ for some $t_{0} \in[0,1]^{2}$,

(iii) $\left\|\sigma_{n}-\sigma\right\| \underset{n \rightarrow \infty}{\stackrel{\text { a.s. }}{\longrightarrow}} 0$.

If any of the above statements hold true, then also

$$
\text { (iv) }\left\|\widetilde{\sigma}_{n}-\sigma\right\| \underset{n \rightarrow \infty}{\stackrel{\text { a.s. }}{\longrightarrow}} 0 \text {. }
$$


As a straightforward application, Theorems 3 and 4 enable for consistent estimation of extremes of mean functions, covariance, or correlation surfaces, or the integrals of them, for both complete and dense functional data. For covariance surfaces, such functionals generalize the multivariate dispersion measures commonly used in statistical analysis. To illustrate this method of estimation of tensor surfaces, consider the following two examples.

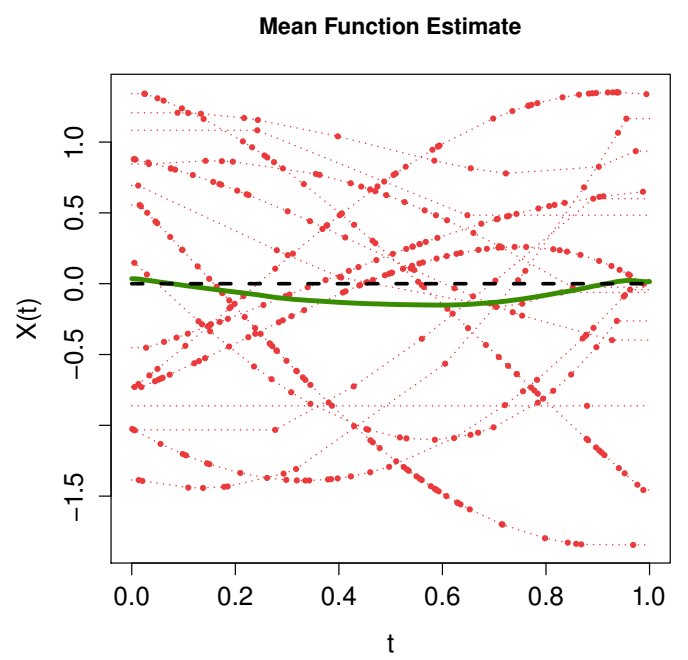

(A)

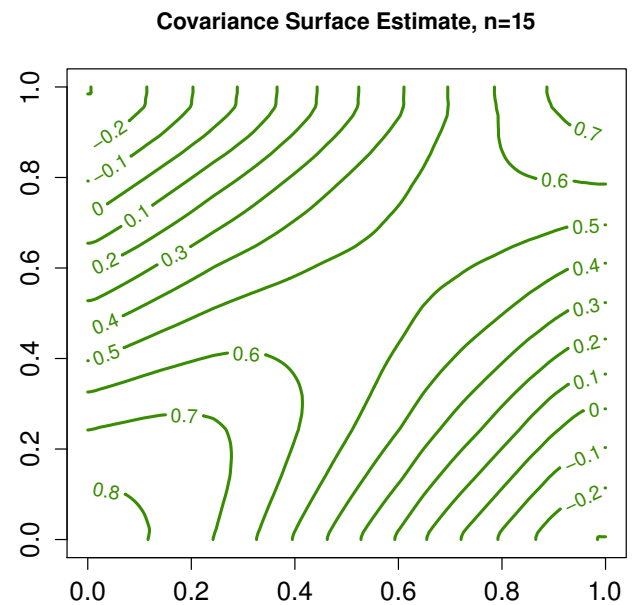

(C)

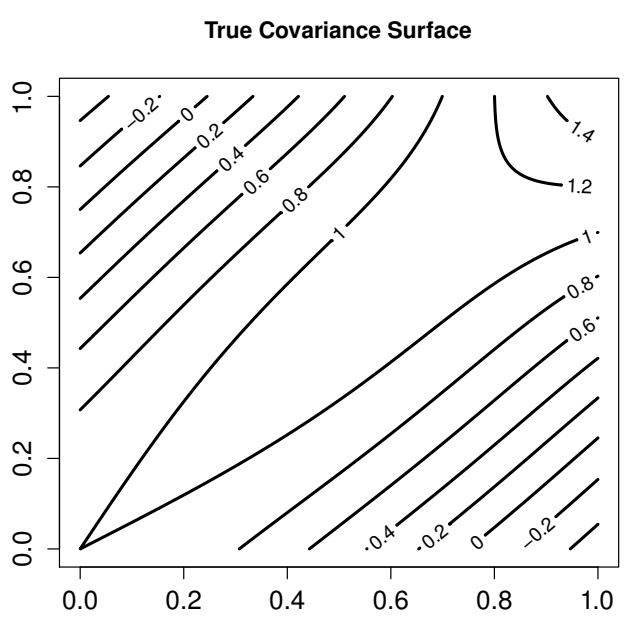

(B)

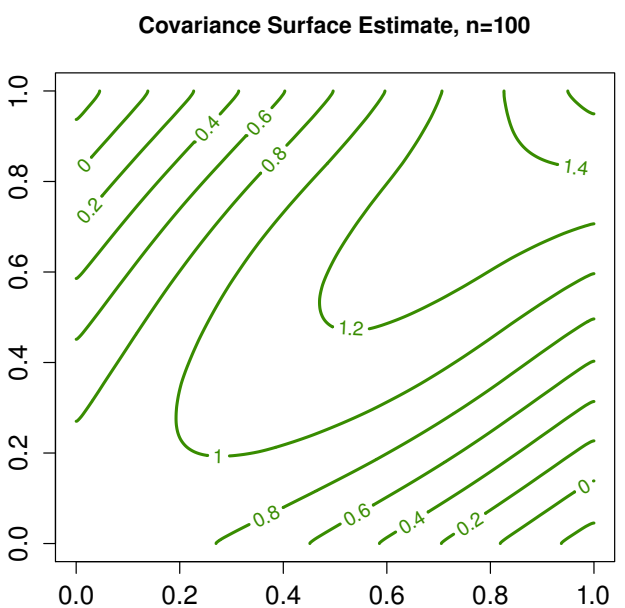

(D)

Figure 1. Simulated data example: (A) A random sample of 15 piecewise linear reconstructions of discretely observed functions (dotted lines) along with their observation points (brown dots), and the associated piecewise linear estimate (solid green line) of the mean function $\mathrm{E}[X] \equiv 0$ (dashed horizontal line); (B) The true covariance surface $\sigma$ of the random function $X$, and its bilinear estimates $\widetilde{\sigma}_{n}$ based on $(C) n=15$, and $(D) n=100$ sampled random functions. 
4.1. Simulated Data Example. Let $X_{1}, \ldots, X_{n}$ be a random sample from $X \sim P \in$ $\mathcal{P}(\mathcal{C}([0,1]))$ defined by

$$
X(t)=A \cos (2 \pi t / 3)+B \sin (5 \pi t / 7)+C\left(1-\exp \left(t^{2}\right)\right) / 2 \quad \text { for } t \in[0,1],
$$

where $A, B$ and $C$ are independent standard normal random variables. For $i=1, \ldots, n$, $X_{i}$ is observed at $M_{i}=\left\lfloor i^{3 / 2}\right\rfloor$ points uniformly distributed over [0,1], independent of $A, B$ and $C$. In Fig. $1 \mathrm{~A}$ we have one such random sample of $n=15$ curves, with the corresponding piecewise linear estimate $1 / n \sum_{i=1}^{n} \widetilde{X}_{i}$ of the mean function $\mathrm{E}[X] \equiv 0$ based on the reconstructions (6) of the sample curves. In Fig. 1B we see the contour plot of the true covariance surface $\sigma$ of $X$, and in Fig. 1C and 1D are its bilinear estimates $\widetilde{\sigma}_{n}$ for $n=15$ and $n=100$, respectively. We see that while $n=15$ allows for a good estimation of the mean curve, a larger sample size is needed for reliable estimation of higher-dimensional tensor surfaces of $X$.

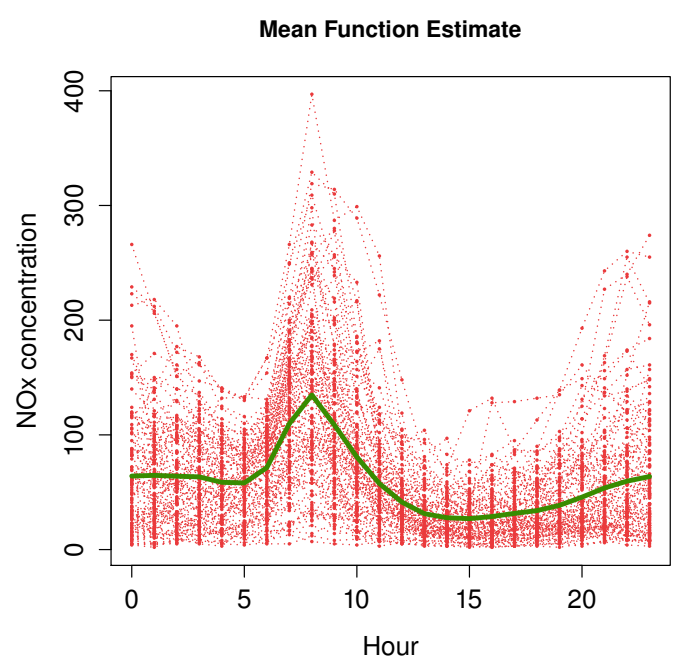

(A)

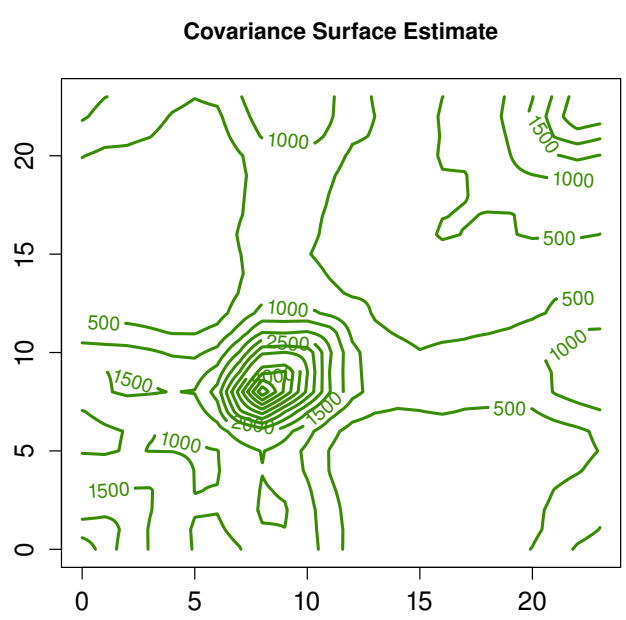

(B)

FiguRE 2. Real data example: (A) The piecewise linear reconstructions of discretely observed functions (dotted lines) along with their observation points (brown dots), and the associated piecewise linear estimate (solid green line) of the mean function; $(B)$ the bilinear estimate $\widetilde{\sigma}_{n}$ of the covariance surface $\sigma$ based on the $n=115$ discretely observed curves.

4.2. Real Data Example. Consider the Poblenou data provided in the package fda .usc in $\mathrm{R}$ (Febrero-Bande and Oviedo de la Fuente, 2012). Each curve $X_{i}, i=1, \ldots, 115$ corresponds to one day in the year 2005, and consists of $M_{i}=24$ hourly measurements of mono-nitrogen oxide levels, measured at an air quality monitoring station in Barcelona. In Fig. 2A we see the measured data points and their interpolations (6) forming the functional dataset. The mean function estimated from the functional data is in fact the linear interpolant of the mean values of the observed values at the 24 observation points. In Fig. 2B the covariance surface estimated from these functions is displayed. Despite a limited number of observation points per curve, and the regularity of the grid of observed points, the resulting estimate appears to be rather flexible, and provides a reasonably 
accurate description of the data. Obviously though, the multi-linear estimates of tensor products are typically not smooth. Smooth surfaces can be obtained straightforwardly by employing a more involved univariate interpolation technique, such as higher order splines, to obtain smooth reconstructed curves $\widetilde{X}_{i}$. Then, one could proceed analogously as here. This comes, however, at the cost of selecting additional tuning parameters in the smoothing process.

\section{Appendix: Proofs of the Theoretical Results}

Proof of Lemma 1. For $T$ uniformly distributed over $[0,1]$, the proof follows from the result of Slud (1978, Theorem 2.1), see also Devroye (1981). In general, apply the mean value theorem to the uniformly distributed random variables obtained by inverse transform sampling.

Proof of Lemma 2. For $d=1$, condition (D.3) is satisfied by de Boor (2001, Chapter III). For $d>1$ we follow an argumentation similar to that of Vakarchuk and Myskin (2005). Assume, without loss of generality, that for each involved random function $X$ observed at $\mathcal{T}$ we have $T_{1}=0$ and $T_{M}=1$. The corresponding interpolant function (6) can then be expressed in basis expansion form, more suitable for extensions to tensor product surfaces. For $t \in[0,1]$ and $j=1, \ldots, M-1$ define a basis of piecewise linear functions

$$
H_{0, j}(t)=\left\{\begin{array}{ll}
\frac{T_{j+1}-t}{T_{j+1}-T_{j}} & \text { if } t \in\left[T_{j}, T_{j+1}\right), \\
0 & \text { otherwise, }
\end{array} \quad H_{1, j}(t)= \begin{cases}\frac{t-T_{j}}{T_{j+1}-T_{j}} & \text { if } t \in\left[T_{j}, T_{j+1}\right), \\
0 & \text { otherwise }\end{cases}\right.
$$

Function $\widetilde{X}$ can then be written as

$$
\tilde{X}(t)=\sum_{j=1}^{M-1} \sum_{k=0}^{1} X\left(T_{j+k}\right) H_{k, j}(t) \text { for all } t \in[0,1) .
$$

Here, for each $t \in\left[T_{j}, T_{j+1}\right)$ only the two summands corresponding to the index $j$ may attain non-zero values. This simplifies the above formula to

$$
\widetilde{X}(t)=\sum_{k=0}^{1} X\left(T_{j+k}\right) H_{k, j}(t) \text { for } t \in\left[T_{j}, T_{j+1}\right) .
$$

The multi-linear interpolant of the tensor product surface $X \otimes X$ can then be written in the form

$$
\widetilde{X} \otimes \widetilde{X}(t)=\sum_{k=0}^{1} \sum_{k^{\prime}=0}^{1} X\left(T_{j+k}\right) H_{k, j}\left(t_{1}\right) X\left(T_{j^{\prime}+k^{\prime}}\right) H_{k^{\prime}, j^{\prime}}\left(t_{2}\right)
$$

for $t=\left(t_{1}, t_{2}\right) \in\left[T_{j}, T_{j+1}\right) \times\left[T_{j^{\prime}}, T_{j^{\prime}+1}\right)$. The difference of the original tensor product and its approximation at arbitrary $t \in[0,1)^{2}$ as above can then be expressed as

$$
\begin{aligned}
|\widetilde{X} \otimes \widetilde{X}(t)-X \otimes X(t)| & =\left|\sum_{k=0}^{1} \sum_{k^{\prime}=0}^{1} H_{k, j}\left(t_{1}\right) H_{k^{\prime}, j^{\prime}}\left(t_{2}\right)\left(X\left(T_{j+k}\right) X\left(T_{j^{\prime}+k^{\prime}}\right)-X\left(t_{1}\right) X\left(t_{2}\right)\right)\right| \\
& \leq \sum_{k=0}^{1} \sum_{k^{\prime}=0}^{1} H_{k, j}\left(t_{1}\right) H_{k^{\prime}, j^{\prime}}\left(t_{2}\right)\left|X\left(T_{j+k}\right) X\left(T_{j^{\prime}+k^{\prime}}\right)-X\left(t_{1}\right) X\left(t_{2}\right)\right| \\
& \leq \mathcal{M}(X \otimes X, \sqrt{2} \nu(\mathcal{T})),
\end{aligned}
$$


immediately yielding condition (D.3) for $d=2, C=\sqrt{2}$, and $D=1$. Analogously, for any $d \in \mathbb{N}$ it can be shown that for the piecewise multi-linear interpolation of the $d$ th tensor product surface the condition (D.3) is satisfied for $C=\sqrt{d}$ and $D=1$.

Auxiliary Results needed for proving Theorem 1. The following lemmas will be used in the proof of the SLLN stated in Theorem 1. The first one provides various characterizations of the moment continuity property.

Lemma 3. Let $d \in \mathbb{N}$ and $X \sim P \in \mathcal{P}(\mathcal{C}([0,1]))$. Then the following statements are equivalent

(i) $X \in \mathcal{M C}_{d}$,

(ii) for each $t \in[0,1]^{d}$ there exists $\delta=\delta(t)>0$ such that

$$
\mathrm{E}\left[\sup _{s:\|s-t\|_{E}<\delta}\left|\bigotimes_{j=1}^{d} X(s)-\bigotimes_{j=1}^{d} X(t)\right|\right]<\infty
$$

(iii) it holds true that

$$
\lim _{\delta \rightarrow 0+} \mathrm{E}\left[\mathcal{M}\left(\bigotimes_{j=1}^{d} X, \delta\right)\right]=0
$$

Proof. $(i) \Longleftrightarrow(i i)$. For $d=1$, see Hansen (2012, Lemma 2.1). The general case is analogous.

$($ iii $) \Longrightarrow(i)$. Trivial.

$((i)$ and $(i i)) \Longrightarrow(i i i)$. Designate $Y=\bigotimes_{j=1}^{d} X$, and for each $t \in[0,1]^{d}$ write for $\delta \leq \sqrt{d}$

$$
\delta_{t}^{*}=\sup \left\{\delta / 2: \mathrm{E}\left[\sup _{s:\|s-t\|_{E}<\delta}|Y(s)-Y(t)|\right]<\infty\right\} .
$$

By Parts $(i)$ and $(i i)$ we know that $\delta_{t}^{*}>0$ for any $t \in[0,1]^{d}$. Denote $\delta^{*}=\inf _{t \in[0,1]^{d}} \delta_{t}^{*}$.

Now we show that $\delta^{*}>0$. To see this, note first that by (8) we have for any $t \in[0,1]^{d}$

$$
\mathrm{E}\left[\sup _{s:\|s-t\|_{E}<\delta_{t}^{*}}|Y(s)-Y(t)|\right]<\infty
$$

and at the same time

$$
\mathrm{E}\left[\sup _{s:\|s-t\|_{E}<4 \delta_{t}^{*}}|Y(s)-Y(t)|\right]=\infty \text { provided that } 4 \delta_{t}^{*}<\sqrt{d} .
$$

Assume now for contradiction that for some sequence $\left\{t_{n}\right\}_{n=1}^{\infty} \subset[0,1]^{d}$ the convergence $\delta_{t_{n}}^{*} \underset{n \rightarrow \infty}{\longrightarrow} 0$ takes place. By compactness of $[0,1]^{d}$, we may without loss of generality assume that $t_{n} \underset{n \rightarrow \infty}{\longrightarrow} t_{\infty}$ for some $t_{\infty} \in[0,1]^{d}$. Using Part $(i i), \delta_{t_{\infty}}^{*}>0$. Find $n \in \mathbb{N}$ large enough so that $\left\|t_{n}-t_{\infty}\right\|_{E}<\delta_{t_{\infty}}^{*} / 2$ and $4 \delta_{t_{n}}^{*}<\delta_{t_{\infty}}^{*} / 2$. Then $\mathcal{U}\left(t_{n}, 4 \delta_{t_{n}}^{*}\right) \subseteq \mathcal{U}\left(t_{\infty}, \delta_{t_{\infty}}^{*}\right)$, 
where $\mathcal{U}(t, \varepsilon)$ is the open ball in $[0,1]^{d}$ around $t$ having radius $\varepsilon$. By (9) for $t=t_{\infty}$

$$
\begin{aligned}
\mathrm{E}\left[\sup _{s:\left\|s-t_{n}\right\|_{E}<4 \delta_{t_{n}}^{*}}\left|Y(s)-Y\left(t_{n}\right)\right|\right] & \leq \mathrm{E}\left[\sup _{s:\left\|s-t_{n}\right\|_{E}<4 \delta_{t_{n}}^{*}}\left(\left|Y(s)-Y\left(t_{\infty}\right)\right|+\left|Y\left(t_{\infty}\right)-Y\left(t_{n}\right)\right|\right)\right] \\
& \leq 2 \mathrm{E}\left[\sup _{s:\left\|s-t_{\infty}\right\|_{E}<\delta_{t_{\infty}}^{*}}\left|Y(s)-Y\left(t_{\infty}\right)\right|\right]<\infty .
\end{aligned}
$$

This contradicts either with (10) if $\delta_{t_{n}}^{*}<\sqrt{d} / 4$ for some $n \in \mathbb{N}$ large enough, or with the convergence of the sequence $\left\{\delta_{t_{n}}^{*}\right\}_{n=1}^{\infty}$ if $\delta_{t_{n}}^{*} \geq \sqrt{d} / 4$ for all $n \in \mathbb{N}$.

Knowing that $\delta^{*}>0$, consider $\delta$ such that $0<\delta<\delta^{*} / 3$. Let $m(\delta) \in \mathbb{N}$ be such that there exists a set $\left\{u_{1}, \ldots, u_{m(\delta)}\right\} \subset[0,1]^{d}$ so that for any $t \in[0,1]^{d}$ for some $u_{t} \in$ $\left\{u_{1}, \ldots, u_{m(\delta)}\right\}$ we have $\left\|t-u_{t}\right\|_{E}<\delta$. As $[0,1]^{d}$ is compact, we can choose $m(\delta)<\infty$. For any $s, t \in[0,1]^{d},\|s-t\|_{E}<\delta$ we then have

$$
\max \left\{\left\|s-u_{s}\right\|_{E},\left\|t-u_{t}\right\|_{E},\left\|u_{s}-u_{t}\right\|_{E}\right\}<\delta^{*} .
$$

Now we can write

$$
\begin{aligned}
& \mathrm{E}[\mathcal{M}(Y, \delta)]=\mathrm{E}\left[\sup _{s, t:\|s-t\|_{E}<\delta}|Y(s)-Y(t)|\right] \\
& \leq 2 \mathrm{E}\left[\sup _{s:\left\|s-u_{s}\right\|_{E}<\delta}\left|Y(s)-Y\left(u_{s}\right)\right|\right]+\mathrm{E}\left[\sup _{s, t:\|s-t\|_{E}<\delta}\left|Y\left(u_{s}\right)-Y\left(u_{t}\right)\right|\right] \\
& \quad \leq 2 \max _{j=1, \ldots, m(\delta)} \mathrm{E}\left[\sup _{s:\left\|s-u_{j}\right\|_{E}<\delta^{*}}\left|Y(s)-Y\left(u_{j}\right)\right|\right]+\mathrm{E}\left[\max _{\substack{i, j=1, \ldots ., m(\delta) \\
\left\|u_{i}-u_{j}\right\|_{E}<\delta^{*}}}\left|Y\left(u_{i}\right)-Y\left(u_{j}\right)\right|\right] \\
& \quad \leq(2+m(\delta)) \max _{j=1, \ldots, m(\delta)} \mathrm{E}\left[\sup _{s:\left\|s-u_{j}\right\|_{E}<\delta^{*}}\left|Y(s)-Y\left(u_{j}\right)\right|\right]<\infty .
\end{aligned}
$$

The first inequality on the bottom line of the previous display follows from

$$
\begin{aligned}
\mathrm{E}\left[\max _{\substack{i, j=1, \ldots, m(\delta) \\
\left\|u_{i}-u_{j}\right\|_{E}<\delta^{*}}}\left|Y\left(u_{i}\right)-Y\left(u_{j}\right)\right|\right] & \leq \mathrm{E}\left[\sum_{i=1}^{m(\delta)} \max _{\substack{j=1, \ldots, m(\delta) \\
\left\|u_{i}-u_{j}\right\|_{E}<\delta^{*}}}\left|Y\left(u_{i}\right)-Y\left(u_{j}\right)\right|\right] \\
& \leq m(\delta) \max _{j=1, \ldots, m(\delta)} \mathrm{E}\left[\sup _{s:\left\|s-u_{j}\right\|_{E}<\delta^{*}}\left|Y(s)-Y\left(u_{j}\right)\right|\right] .
\end{aligned}
$$

Part $(i i)$ of the lemma now yields (11).

To conclude the proof, note that for any $x \in \mathcal{C}([0,1])$ and $\delta^{\prime}>\delta>0$ we have $\mathcal{M}\left(\bigotimes_{j=1}^{d} x, \delta^{\prime}\right) \geq \mathcal{M}\left(\bigotimes_{j=1}^{d} x, \delta\right)$. Since $\bigotimes_{j=1}^{d} x \in \mathcal{C}\left([0,1]^{d}\right)$ we also know that

$$
\lim _{\delta \rightarrow 0+} \mathcal{M}\left(\bigotimes_{j=1}^{d} x, \delta\right)=0 \text { for any } x \in \mathcal{C}([0,1]) \text {. }
$$

Thus, (11) allows for the application of the dominated convergence theorem and the desired (7) follows. 
Next, we provide a SLLN for stochastically dominated random variables. It presents an essential extension of the Kolmogorov's SLLN for a sequence of independent, but not identically distributed random variables without imposing restrictions on their second moments.

Lemma 4. Let $\left\{Y_{n}\right\}_{n=1}^{\infty}$ be a sequence of independent random variables, $\mathrm{E}\left[\left|Y_{n}\right|\right] \underset{n \rightarrow \infty}{\longrightarrow} 0$ and let $\left\{Z_{n}\right\}_{n=1}^{\infty}$ be a sequence of independent identically distributed random variables such that $\mathrm{E}\left[\left|Z_{1}\right|\right]<\infty$. Let for each $n \in \mathbb{N}$

$$
\mathrm{P}\left(\left|Y_{n}\right|>t\right) \leq \mathrm{P}\left(\left|Z_{n}\right|>t\right) \text { for all } t \geq 0 \text {. }
$$

Then $1 / n \sum_{i=1}^{n} Y_{n} \underset{n \rightarrow \infty}{\stackrel{\text { a.s. }}{\longrightarrow}} 0$.

Proof. The proof proceeds in the same vein as the proof of Kolmogorov's SLLN as derived in Loève (1977, Theorem B in Section 17.3). The only difference that needs to be introduced to the proof in order to deal with stochastically dominated random variables, apart from (12), is a bound of Adler and Rosalsky (1987, Lemma 1)

$$
\mathrm{E}\left[Y_{n}^{2} \mathbb{I}\left(\left|Y_{n}\right| \leq t\right)\right] \leq t^{2} \mathrm{P}\left(\left|Z_{n}\right|>t\right)+\mathrm{E}\left[\left|Z_{n}\right|^{2} \mathbb{I}\left(\left|Z_{n}\right| \leq t\right)\right] \text { for all } t \geq 0
$$

where $\mathbb{I}(A)$ is 1 if $A$ is true, 0 if not.

\section{Proof of Theorem 1.}

$(i) \Longrightarrow(i i)$. This is a trivial consequence of Part (ii) of Lemma 3 - using this, for any $t \in[0,1]^{d}$ and $\delta>0$ small enough we have

$$
\begin{gathered}
\mathrm{E}\left[\sup _{s:\|s-t\|_{E}<\delta}\left|\bigotimes_{j=1}^{d} X(t)-\bigotimes_{j=1}^{d} X(s)\right|\right] \leq \mathrm{E}\left[\left|\bigotimes_{j=1}^{d} X(t)\right|\right]+\mathrm{E}\left[\left\|\bigotimes_{j=1}^{d} X\right\|\right]<\infty, \\
\mathrm{E}\left[\left|\bigotimes_{j=1}^{d} X(t)\right|\right] \leq \mathrm{E}\left[\left\|\bigotimes_{j=1}^{d} X\right\|\right]<\infty .
\end{gathered}
$$

(ii) $\Longrightarrow\left(\right.$ i). $X \in \mathcal{M C}_{d}$ implies, by Part (iii) of Lemma 3, that for some $\delta>0$ small enough (independent of $t \in[0,1]$ ) we can write

$$
\mathrm{E}\left[\left\|\bigotimes_{j=1}^{d} X\right\|\right] \leq \mathrm{E}\left[\left|X\left(t_{0}\right)\right|\right]+N(\delta) \mathrm{E}\left[\mathcal{M}\left(\bigotimes_{j=1}^{d} X ; \delta\right)\right]<\infty,
$$

where $N(\delta)$ is the covering number of the set $[0,1]^{d}$ by balls with radius $\delta / 2$.

$(i) \Longleftrightarrow($ iii $)$. See Ledoux and Talagrand (2011, Corollary 7.10).

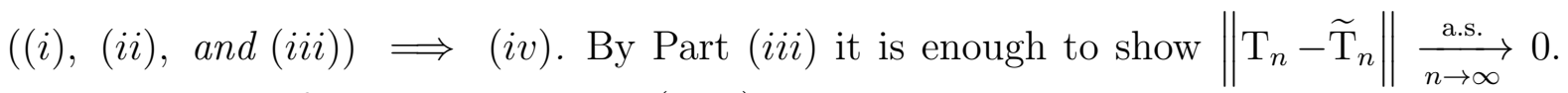
This will be verified using condition (D.3) by bounding

$$
\left\|\mathrm{T}_{n}-\widetilde{\mathrm{T}}_{n}\right\| \leq \frac{1}{n} \sum_{i=1}^{n} \sup _{t \in[0,1]^{d}}\left|\bigotimes_{j=1}^{d} X_{i}(t)-\bigotimes_{j=1}^{d} \widetilde{X}_{i}(t)\right| \leq \frac{1}{n} \sum_{i=1}^{n} D \mathcal{M}\left(\bigotimes_{j=1}^{d} X_{i}, C \nu\left(\mathcal{T}_{i}\right)\right) .
$$


Let us now inspect the sequence $\left\{\mathcal{M}\left(\bigotimes_{j=1}^{d} X_{n}, \nu\left(\mathcal{T}_{n}\right)\right)\right\}_{n=1}^{\infty}$ (taking $C=1$, without loss of generality). These random variables are independent of each other, and for their expectations we can write by Part (iii) of Lemma 3 and Part (ii) of the present theorem

$$
\lim _{n \rightarrow \infty} \mathrm{E}\left[\mathcal{M}\left(\bigotimes_{j=1}^{d} X_{n}, \nu\left(\mathcal{T}_{n}\right)\right)\right]=0
$$

Moreover, for any $n \in \mathbb{N}$, we can introduce a simple stochastic bound

$$
\mathcal{M}\left(\bigotimes_{j=1}^{d} X_{n}(\omega), \nu\left(\mathcal{T}_{n}(\omega)\right)\right) \leq \mathcal{M}\left(\bigotimes_{j=1}^{d} X_{n}(\omega), \sqrt{d}\right) \text { for all } \omega \in \Omega
$$

Here, $\left\{\mathcal{M}\left(\bigotimes_{j=1}^{d} X_{n}, \sqrt{d}\right)\right\}_{n=1}^{\infty}$ is already a sequence of independent, and identically distributed random variables with expectation

$$
\mathrm{E}\left[\mathcal{M}\left(\bigotimes_{j=1}^{d} X, \sqrt{d}\right)\right]=\mathrm{E}\left[\sup _{s, t:\|s-t\|_{E}<\sqrt{d}}\left|\bigotimes_{j=1}^{d} X(s)-\bigotimes_{j=1}^{d} X(t)\right|\right] \leq 2 \mathrm{E}\left[\left\|\bigotimes_{j=1}^{d} X\right\|\right]
$$

where the last term is finite by Part $(i)$. The desired conclusion then follows directly from Lemma 4.

Proof of Theorem 2. Suppose that $\mathrm{E}\left[\left\|\bigotimes_{j=1}^{d^{\prime}} X\right\|\right]=\infty$, i.e. $\sum_{n=1}^{\infty} \mathrm{P}\left(A_{n}\right)=\infty$, where

$$
A_{n}=\left\{\omega \in \Omega:\left\|\bigotimes_{j=1}^{d^{\prime}} X(\omega)\right\| \geq n\right\}
$$

For any $\omega \in A_{n}$ we have

$$
\left\|\bigotimes_{j=1}^{d} X(\omega)\right\|=\sup _{\substack{t_{j} \in[0,1] \\ j=1, \ldots, d}}\left|\prod_{j=1}^{d} X\left(t_{j}, \omega\right)\right|=\left(\sup _{t \in[0,1]}|X(t, \omega)|\right)^{d}=\left(\|X(\omega)\|^{d^{\prime}}\right)^{d / d^{\prime}} \geq n^{d / d^{\prime}} \geq n
$$

providing the desired

$$
\sum_{n=1}^{\infty} \mathrm{P}\left(\left\|\bigotimes_{j=1}^{d} X\right\| \geq n\right) \geq \sum_{n=1}^{\infty} \mathrm{P}\left(A_{n}\right)=\infty
$$

meaning that $\mathrm{E}\left[\left\|\bigotimes_{j=1}^{d} X\right\|\right]=\infty$.

Acknowledgement. This research is supported by the IAP research network no. P7/06 of the Federal Science Policy (Belgium). The first author is a Research Assistant of the Research Foundation - Flanders, and acknowledges support from this foundation. The second author gratefully acknowledges support from the GOA/12/014-project of the Research Fund KU Leuven. 


\section{REFERENCES}

Abramowicz, K. and Seleznjev, O. (2013). Piecewise-multilinear interpolation of a random field. Adv. in Appl. Probab., 45(4):945-959.

Adler, A. and Rosalsky, A. (1987). Some general strong laws for weighted sums of stochastically dominated random variables. Stochastic Anal. Appl., 5(1):1-16.

Cai, T. T. and Yuan, M. (2011). Optimal estimation of the mean function based on discretely sampled functional data: phase transition. Ann. Statist., 39(5):2330-2355.

de Boor, C. (2001). A Practical Guide to Splines, volume 27 of Applied Mathematical Sciences. Springer-Verlag, New York, revised edition.

Devroye, L. (1981). Laws of the iterated logarithm for order statistics of uniform spacings. Ann. Probab., 9(5):860-867.

Febrero-Bande, M. and Oviedo de la Fuente, M. (2012). Statistical computing in functional data analysis: The R package fda.usc. Journal of Statistical Software, 51(4):1-28.

Ferraty, F. and Vieu, P. (2006). Nonparametric Functional Data Analysis. Theory and Practice. Springer Series in Statistics. Springer, New York.

Hansen, L. P. (1982). Large sample properties of generalized method of moments estimators. Econometrica, 50(4):1029-1054.

Hansen, L. P. (2012). Proofs for large sample properties of generalized method of moments estimators. J. Econometrics, 170(2):325-330.

Klimke, A. and Wohlmuth, B. (2005). Algorithm 847: spinterp: piecewise multilinear hierarchical sparse grid interpolation in MATLAB. ACM Trans. Math. Software, 31(4):561-579.

Ledoux, M. and Talagrand, M. (2011). Probability in Banach Spaces. Isoperimetry and Processes. Classics in Mathematics. Springer-Verlag, Berlin. Reprint of the 1991 edition.

Li, Y. and Hsing, T. (2010). Uniform convergence rates for nonparametric regression and principal component analysis in functional/longitudinal data. Ann. Statist., 38(6):3321-3351.

Loève, M. (1977). Probability Theory I. Springer-Verlag, New York-Heidelberg, fourth edition. Graduate Texts in Mathematics, Vol. 45.

Nagy, S., Gijbels, I., and Hlubinka, D. (2016). Weak convergence of discretely observed functional data with applications. Journal of Multivariate Analysis, 146:46 - 62. Special Issue on Statistical Models and Methods for High or Infinite Dimensional Spaces.

Ramsay, J. O. and Silverman, B. W. (2005). Functional Data Analysis. Springer Series in Statistics. Springer, New York, Second edition.

Slud, E. (1978). Entropy and maximal spacings for random partitions. Z. Wahrscheinlichkeitstheorie und Verw. Gebiete, 41(4):341-352.

Vakarchuk, S. B. and Myskin, K. Y. (2005). Some problems of the simultaneous approximation of functions of two variables and their derivatives by interpolation bilinear splines. Ukraïn. Mat. Zh., 57(2):147-157.

Varadarajan, V. S. (1958). On the convergence of sample probability distributions. Sankhyā, 19:23-26. 